\section{Assessment of serum level cholinesterase as a biomarker of liver cirrhosis in Egyptian cirrhotic patients}

\author{
Mona A. Amin, ${ }^{1}$ \\ Marwa E. El-Shahat, ${ }^{1}$ \\ Nouman EI-Garem, ${ }^{1}$ \\ Ahmed Soliman, ${ }^{1}$ Eman Obaia ${ }^{2}$ \\ ${ }^{1}$ Department of Internal Medicine and \\ ${ }^{2}$ Department of Biochemistry, Faculty of \\ Medicine, Cairo University, Egypt
}

\begin{abstract}
Serum cholinesterase levels are closely correlated with the severity of liver disease. The aim of the paper was to assess the value of serum cholinesterase in evaluating liver reserve function in cirrhotic patients. 90 patients with liver cirrhosis and thirty healthy control group were included. Liver cirrhosis patients were classified according to child score into three equal groups: Child A liver cirrhosis, Child B liver cirrhosis and Child C liver cirrhosis. Patients were subjected to clinical evaluation, laboratory analysis, abdominal U/S. Measuring serum cholinesterase, and Calculation of both Child and model of end stage liver disease (MELD) scores. The level of serum cholinesterase was higher in control group than the three groups of liver cirrhosis with median (IQR) 17,410 (12,111-21,774), 7528 (5200-9856), 6021 (4500-7542), 3828.5 (1541-6060), respectively $\mathrm{P}<0.001$ ). And the level of serum cholinesterase was higher in Child A more than Child B and Child $C$ and the level of serum cholinesterase was higher in Child B more than Child $\mathrm{C}$ with very strong negative correlation between serum Cholinesterase level and Child score $(\mathrm{r}=-0.9, \mathrm{P}<0.001)$. Also strong negative correlation between serum Cholinesterase level and MELD score $(\mathrm{r}=-$ $0.85, \mathrm{P}=0.001$ ), and positive correlation with prothrombin concentration $(\mathrm{r}=0.554$, $\mathrm{P}=0.009$ ), and serum albumin levels $(r=0.582, P=0.0002)$. Serum cholinesterase is a good biomarker of cirrhosis. Since it distinguishes decompensated from compensated cirrhosis well, low levels in cirrhosis may serve as a useful prognostic marker of advanced liver disease.
\end{abstract}

\section{Introduction}

According to the World Health
Organization, about 800,000 people die of cirrhosis annually. In the United States, cirrhosis accounts for about 27,500 deaths each year, or a death rate of 9.3 per 100,000 , making it the 12th leading cause of death overall. ${ }^{1}$ Egypt has the highest burden of $\mathrm{HCV}$ infection worldwide, the prevalence was estimated about $10 \%$ of chronic $\mathrm{HCV}$ infection among persons aged 15-59 years. ${ }^{2}$

The prognosis of cirrhosis is highly variable since it is influenced by many factors, including etiology, severity, presence of complications, and comorbid diseases. Multiple studies have attempted to predict the prognosis of patients with cirrhosis based upon clinical and laboratory information. One of the earliest models (the Child's classification) continues to be a useful method for stratifying disease severity, surgical risk, and overall prognosis.

There are several limitations for The Child-Turcotte-Pugh (CTP) Score. It has limited discriminatory capacity and does not adequately segregate patients with progressively abnormal lab results. Also, two of the five parameters (encephalopathy and ascites) must be subjectively interpreted. In addition, variceal hemorrhage, which is an important marker of hepatic decompensation, is not included in the definition of CTP class. $^{3}$

The model of end stage liver disease (MELD) score is developed to estimate the risks in patients undergoing trans-jugular portosystemic shunt procedure and to attribute the risk of death for patients with liver cirrhosis, rendering it the leading parameter for allocation of liver transplantation. ${ }^{4}$

The MELD score may be influenced by the method in which serum creatinine and INR are measured across laboratories. The MELD score has fewer limitations than the CTP score because it uses only objective data and can distinguish disease severity along a continuous spectrum. Furthermore the MELD score was developed for prediction of short term survival within three months, while the prediction for the survival of more than three months is much weaker. ${ }^{5}$ Therefore the evaluation of new markers is an important task in patients with liver cirrhosis

Cholinesterase is a term that refers to two enzymes; Acetylcholinesterase (also known as RBC cholinesterase or true cholinesterase) and Pseudocholinesterase (also known as plasma cholinesterase). Both of them catalyze the hydrolysis of the neurotransmitter acetylcholine into choline and acetic acid. ${ }^{6}$

Cholinesterase has a serum half-life between 8 and 12 days, and is an alpha 2 globulin, a tetramer of 342,000 molecular weight, that exists in aggregate form.
Correspondence: Mona Ahmed Amin, Department of Internal Medicine and Hepatogastroenterology, Faculty of Medicine, Cairo University, Cairo, Egypt.

E-mail: monasleman@hotmail.com

Key words: liver cirrhosis, cholinesterase, biomarkers.

Contributions: the authors contributed equally.

Conflict of interest: the authors declare no potential conflict of interest.

Received for publication: 2 October 2016 Accepted for publication: 23 January 2017.

This work is licensed under a Creative Commons Attribution NonCommercial 4.0 License (CC BY-NC 4.0).

(C) Copyright M.A. Amin et al., 2017

Licensee PAGEPress, Italy

Gastroenterology Insights 2017; 8:6914

doi:10.4081/gi.2017.6914

Pseudocholinesterase is synthesized in the liver, pancreas, heart and brain white matter, while acetylcholinesterase (true cholinesterase) is found in the RBC's and CSF. It is essential for the degradation of acetylcholine, after impulse transmission has been mediated. ${ }^{7,8}$

The sensitivity and specificity of serum cholinesterase were estimated about 90\% and $100 \%$ respectively by Gokani et al. ${ }^{8}$ and they conclude that it must be added as a routine diagnostic test beside other liver function tests for investigation of liver dysfunction. The estimation of serum cholinesterase reflects hepatocellular damage and is regarded as a sensitive indicator of liver diseases. ${ }^{9}$

\section{Aim of work}

In this work we aimed to assess the value of serum cholinesterase in evaluating liver reserve function in a group of Egyptian cirrhotic patients with different Child score classification.

\section{Materials and Methods}

After ethical approval of the internal medicine ethical committee and informed written consent were obtained from patients we prospectively enrolled 30 sex and age matched normal healthy persons and 90 patients with liver cirrhosis, who were referred to the outpatient department and 
internal medicine hospital at Kasr Al-Ainy between December 2014 and May 2015 .

Patients with liver cirrhosis were divided equally according to Child Score into three groups: Group 1 Child A patients, group 2 Child B patients and group 3 Child C score liver cirrhosis patients .

We excluded patients less than 18 years old , those with history of albumin or blood transfusion four weeks prior to enrolment, history or evidence of variceal bleeding at enrolment, history or evidence of hepatocellular carcinoma on the basis on ultrasonography, $\alpha$-fetoprotoein levels $400 \mathrm{~g} / \mathrm{L}$ and or histology at entry and those who underwent Liver transplantation.

The allocated cases in this study were subjected to the: A) full history and clinical evaluation; B) laboratory investigations including: complete blood count (CBC), liver function tests (alanine aminotransferase, aspartate aminotransferase, total bilirubin, direct bilirubin, serum albumin, PT, PC and INR), kidney function test (urea, creatinine, $\mathrm{Na}$ and $\mathrm{K}$ ), serum cholinesterase; C) abdominal ultrasonography.

The variables of the study are: i) ChildPugh score; ii) MELD score; iii) serum cholinesterase.

Blood samples were collected with minimal venostasis. Serum was obtained from clotted blood by centrifugation within $1 \mathrm{~h}$ of sampling. Serum cholinesterase activity was determined using an assay with butyrylthiocholine as the substrate.

\section{Statistical analysis}

Descriptive statistical are described in term of mean, standard deviation, and percentage. For comparing categorical data $\mathrm{Chi}$ square test performed. The data analyzed by using SPSS (Statistical Package for the social science) version 20 for Microsoft Windows. Statistical differences among groups were tested by using independent one way ANOVA test (analysis of variance) for repeated measurements followed by post-hoc comparison with Tukey HSD test. Bonferroni correlation was used when multiple subgroup comparisons were performed. P-value 0.05 was considered to be significant.

\section{Results}

The mean age was 53 years for control and not significantly different from other three groups with child A, B and C (mean; 56, 54 and 56 years respectively). Male sex was representing the majority in all groups. As regard history and clinical data there was statistical significance difference as regard history of $\mathrm{HCV}$ infection, $\mathrm{HBV}$ infection between control and other three groups as shown in Table 1.

There were statistically significant difference as regard AST, ALT $(\mathrm{P}<0.001)$ ,serum albumin level $(\mathrm{P}<0.001)$, total bilirubin level $(\mathrm{P}<0.001), \mathrm{PC}, \mathrm{PT}$ and INR $(\mathrm{P}<0.001)$ serum creatinine $(\mathrm{P}<0.001)$, and serum urea level $(\mathrm{P}<0.001)$ between control group and the other three groups (Table 2).

There was statistically significant lower levels of serum cholinesterase in Child A group compared to the control group $(\mathrm{P}<0.001)$ as well as lower levels of cholinesterase in Child B group compared to the control group $(\mathrm{P}<0.001)$, also cholinesterase level was lower in Child $\mathrm{C}$ group compared to control group $(\mathrm{P}<0.001)$ (Table 3).

There was statistically significant positive correlation between serum cholinesterase level with Albumin, PC and $\mathrm{Na}$ levels $[\mathrm{r}=0.58,0.55,0.28$ respectively $(\mathrm{P}<0.05)]$. while there was statistically significant positive correlation between the serum cholinesrase with INR, serum creatinine, urea and TCL levels $[\mathrm{r}=-0.5,-0.33$,0.35 and 0.02 respectively $(\mathrm{P}<0.05)]$ (Table 4). The correlation studies between serum cholinesterase level and both MELD and Child scores shown in Table 5. It revealed

Table 1. Comparison among the studied groups regarding demographic features and clinical data.

\begin{tabular}{|c|c|c|c|c|c|}
\hline & Control & Child A & Child B & Child C & P value \\
\hline Age $($ mean \pm SD) & $53.6 \pm 9.6$ & $56.2 \pm 9.6$ & $54.6 \pm 8.3$ & $56.6 \pm 5.0$ & NS \\
\hline Sex (male), N. (\%) & $20(50.0)$ & $22(55.0)$ & $12(95.0)$ & $19(80.0)$ & $0.01 \mathrm{~S}$ \\
\hline Smoking, (N,\%) & 10 & 14 & 10 & 8 & NS 0.4 \\
\hline HTN (N,\%) & 8 & 2 & 1 & 0 & NS 0.8 \\
\hline DM (N,\%) & 5 & 6 & 4 & 1 & NS 0.2 \\
\hline $\mathrm{HCV}(\mathrm{N}, \%)$ & 0 & 25 & 10 & 13 & S 0.01 \\
\hline HBV (N,\%) & 0 & 5 & 19 & 17 & S 0.02 \\
\hline Bilharziasis (N,\%) & 0 & 0 & 1 & 0 & NS 0.4 \\
\hline
\end{tabular}

HTN, hypertension; DM, diabetes mellitus; HCV, hepatitis C virus; HBV, hepatitis B virus.

Table 2. Comparison among the studied groups regarding liver functions.

\begin{tabular}{lccccc} 
& Control & Child A & Child B & Child C & P value \\
ALT (U/L) & $23 \pm 7$ & $45.7 \pm 20$ & $45.7 \pm 20$ & $46.5 \pm 21.2$ & $<0.001$ \\
AST (U/L) & $30 \pm 5$ & $47.3 \pm 19$ & $50.9 \pm 23$ & $61.2 \pm 22.5$ & $<0.001$ \\
\hline Albumin (mg/dL) & $3.8 \pm 1.5$ & $3.5 \pm 0.4$ & $2.8 \pm 0.5$ & $1.9 \pm 0.6$ & $<0.001$ \\
Total bilirubin (mg/dL) & $0.8 \pm 0.2$ & $1.2 \pm 0.3$ & $1.6 \pm 0.3$ & $5.7 \pm 5.7$ & $<0.001$ \\
\hline PC & $85 \pm 15$ & $76.5 \pm 17.5$ & $53 \pm 10$ & $49.1 \pm 14.3$ & $<0.001$ \\
PT & $12 \pm 1$ & $13.6 \pm 1.9$ & $15 \pm 7$ & $20.3 \pm 4.4$ & $<0.001$ \\
\hline INR & $1.0 \pm 0.2$ & $1.2 \pm 0.2$ & $1.6 \pm 0.3$ & $1.8 \pm 0.8$ & $<0.001$ \\
Urea, mg/dL & $18 \pm 10$ & $26 \pm 15$ & $66.9 \pm 45$ & $130 \pm 41.5$ & $<0.001$ \\
\hline Serum creatinine, $\mathrm{mg} / \mathrm{dL}$ & $0.8 \pm 0.3$ & $1.1 \pm 0.4$ & $1.2 \pm 2.9$ & $2 \pm 3.5$ & $<0.001$
\end{tabular}

ALT, alanine aminotransferase; AST, aspartate aminotransferase; PC, prothrombin concentration; PT, prothrombin time; INR, international normalized ratio. 
strong negative correlation between serum Cholinesterase level and MELD score $(\mathrm{r}=0.85, \mathrm{P}=0.001)$, and very strong negative correlation between serum Cholinesterase level and Child score $(r=0.9, \mathrm{P}<0.001)$ (Figure 1).

\section{Discussion}

Child-Pugh score is used to assess the prognosis of chronic liver disease, mainly cirrhosis. Although originally used to predict mortality during surgery, the ChildPugh score is now used to determine the prognosis as well as the required strength of treatment, and the necessity for liver transplantation. However, the cirrhotic patients, particularly those with Child grades B and $\mathrm{C}$ with ascites or hemorrhagic tendency, are usually treated with albumin or blood transfusion, which may affect the real numerical value for calculating the Child-Pugh score.

The model of end stage liver disease (MELD) score was developed to estimate the risks in patients undergoing (TIPS) procedure and to attribute the risk of death for patients with liver cirrhosis allocated for liver transplantation. ${ }^{4}$ MELD scores fail to predict mortality in about $15 \%$ of patients with end-stage liver disease. Incorporation of additional parameters, including serum sodium level, serum albumin level, glucose intolerance, or APACHE II score, may potentially improve the prognostic accura- cy. ${ }^{10}$ The MELD score does not correlate well with severity of hepatic encephalopathy or ascites because approximately $90 \%$ of patients with clinical hepatic encephalopathy or abnormal EEG and neuropsychometric tests had a MELD score
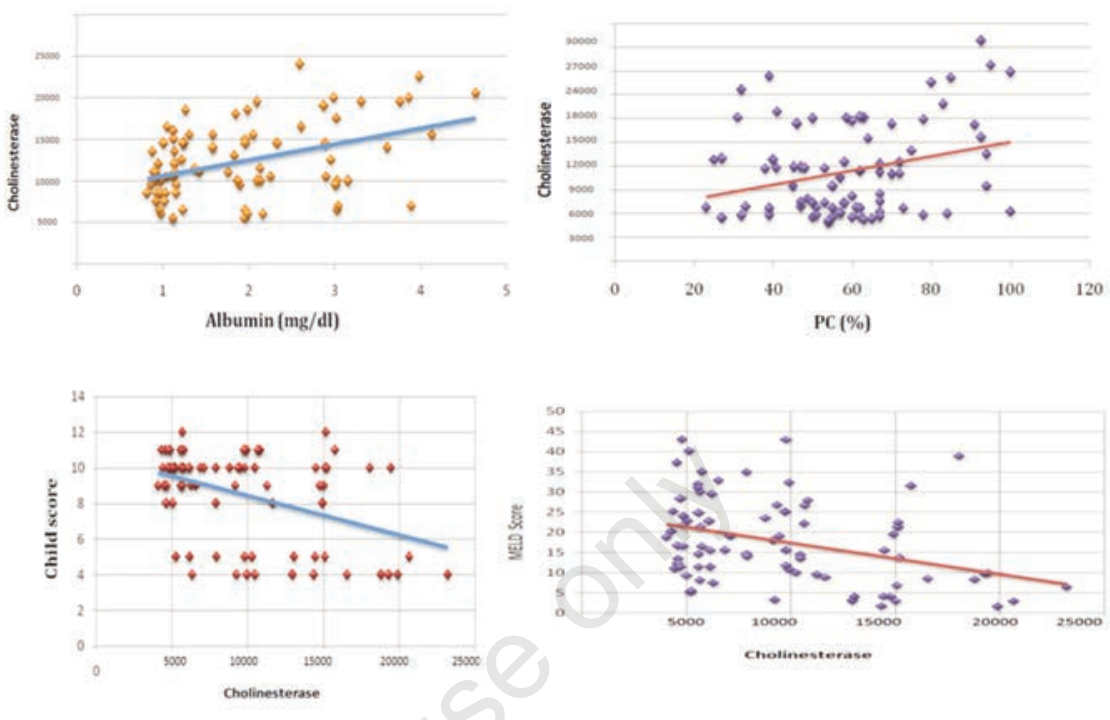

Figure 1. Correlation of cholinesterase and serum albumin, prothrombin concentration, child score and the model of end stage liver disease score.

Table 3. Comparison of serum cholinesterase among control group and liver cirrhosis groups.

\begin{tabular}{|c|c|c|c|c|c|}
\hline & A & B & C & Control & P value \\
\hline Number & 30 & 30 & 30 & 30 & \\
\hline Serum cholinesterase levels, U/L (median range) & $7528(5200-9856)$ & $6021(4500-7542)$ & $3828.5(1541-6060)$ & $17410(12111-21774)$ & $\begin{array}{c}<0.001^{*} \\
<0.001^{* *} \\
<0.001^{* * *}\end{array}$ \\
\hline
\end{tabular}

Table 4. Significant correlations of cholinesterase level with other parameters within the studied groups.

\begin{tabular}{|c|c|c|}
\hline Related pairs, cholinesterase & Correlation coefficient & P value \\
\hline Albumin & 0.582 & $0.0002^{*}$ \\
\hline $\mathrm{PC}$ & 0.554 & $0.009 *$ \\
\hline $\mathrm{Na}$ & 0.278 & $0.008^{*}$ \\
\hline INR & -0.52 & $0.021^{*}$ \\
\hline Creatinine & -0.325 & $0.010^{*}$ \\
\hline Urea & -0.345 & $0.0009 *$ \\
\hline TLC & -0.023 & $0.002 *$ \\
\hline
\end{tabular}

*Significant correlation. PC, prothrombin concentration; Na, serum sodium; INR, international normalized ratio; TLC, total leucocytes count.

Table 5. Correlation between cholinesterase level with model of end stage liver disease (MELD) and child scores within the studied groups.

\begin{tabular}{lcc} 
Related pairs, cholinesterase & P value \\
MELD score & -0.853 & $0.001^{*}$ \\
Child-Pugh score & -0.922 & $<0.001^{*}$ \\
\hline
\end{tabular}

*Significant correlation. 
less than $25 .{ }^{11}$

Biochemical tests for the assessment of liver function (commonly referred to as liver function tests) includes measurement of serum aspartate and alanine transaminases, serum bilirubin, serum albumin, serum protein and alkaline phosphatase, but these tests are often abnormal in patients with clinical problems other than liver dysfunction. ${ }^{12}$

The activities of serum transaminases may be raised due to increased release from non-liver tissue sources in various pathologies. Increased serum alkaline phosphatase activity may result from physiological or pathological enzyme production and release from non-liver tissue sources . Serum bilirubin may be raised because of an increased erythrocyte breakdown rather than because of failure of hepatic clearance. Albumin concentration may be reduced for reasons other than failure of liver synthesis. ${ }^{13}$ As a result, none of these tests can individually confirm liver dysfunction. Therefore there is a need for a test which should be sensitive to liver disease.

The synthesis of cholinesterase is mainly in hepatocytes and it is released into the blood which is reduced in liver dysfunction due to reduced synthesis. ${ }^{8,14}$ The predominant hepatic source of serum cholinesterase, the marked decrease in its synthesis with hepatocyte dysfunction and restoration of synthesis with hepatocyte recovery suggests that serum cholinesterase activity might be a more specific indicator of liver dysfunction than the traditional liver function tests. ${ }^{15}$

Despite all the studies, this simple biochemical test is not routinely done as a liver function test worldwide. In this study, we evaluated serum $\mathrm{ChE}$ as a biomarker of cirrhosis by studying its levels in cirrhotics and controls. We also examined if serum ChE levels can differentiate decompensated cirrhosis (DC) from compensated cirrhosis (CC). We also examined for any correlation between $\mathrm{ChE}$ and other markers of liver function such as serum albumin.

The accumulated evidence suggested that cholinesterase activity is an assessment indicator of liver function in patients with liver disease. In China, cholinesterase has been included in scores to distinguish the hepatitis severity by Society of Liver Disease. ${ }^{16}$

The aim of this work was to assess the value of serum cholinesterase in evaluating liver reserve function in cirrhotic patients. We included 90 patients with liver cirrhosis and divided them into 3 equal groups according to Child-Pugh score and thirty healthy age and sex matched control group to evaluate if the serum level of cholinesterase might be a suitable parameter for disease severity and prognosis in such patients.

In our study, we found cirrhotic to have significantly lower levels of serum $\mathrm{ChE}$ than the control group and the lower levels were correlated with Child Score of such patients.

These results were matched with $\mathrm{Gu}$ et al., ${ }^{17}$ which showed serum cholinesterase tend to decrease significantly in the three grade of cirrhosis: Child A, Child B and Child C.

We also found serum cholinesterase to be higher in compensated cirrhosis than decompensated cirrhosis. These result were matched with the ones of Ramachandran et al., ${ }^{18}$ who found that serum cholinesterase can distinguish between compensated cirrhosis and decompensated cirrhosis.

Serum levels of ChE showed positive correlation with serum albumin levels in our study. These result were matched with Ramachandran et al., ${ }^{18}$ where ChE levels reflect the functional integrity of the liver.

We also found correlation of serum cholinesterase and MELD Score in liver cirrhotic patients, where patients with higher MELD Score have a lower level of cholinesterase. These result were matched with Ramachandran et al. ${ }^{18}$ where correlation coefficient between serum cholinesterase and MELD Score.

\section{Conclusions}

In conclusion, findings of the this study have demonstrated that the level of cholinesterase is correlated with the severity of liver damage and compared with the Child-Pugh score, serum cholinesterase is less complex and not easily affected by treatments for decompensated cirrhosis. The combination of cholinesterase with the Child-Pugh score may be more subjective and accurate in evaluating the liver reserve function of cirrhotic patients.

\section{References}

1. Garcia-Tsao G. Cirrhosis and its sequelae. In: Goldman L, Schafer AI, eds. Goldman's Cecil medicine. 25th ed. Philadelphia: Elsevier Saunders; 2016.

2. El-Zanaty F, Way A. Egypt demographic and health survey 2008. Cairo, Egypt: Ministry of Health, El-Zanaty and Associates, and Macro International: 2009.

3. Thornton K.. Core concepts. Evaluation and prognosis of patients with cirrhosis. Hepatitis C online. 2016. Available from: http://www.hepatitisc.uw.edu/go/ evaluation-staging-monitoring/evaluation-prognosis-cirrhosis/coreconcept/all

4. Kamath PS, Wiesner RH, Malinchoc M, et al. A model to predict survival in patients with end-stage liver disease. Hepatology 2001;33:464-70.

5. Kamath PS, Kim WR. The model for end stage liver disease (MELD). Hepatology 2007;45:797.

6. Brimijoin S, Koenigsberger C. Cholinesterase in neural development: new findings and toxicological implications. Environ Health Perspect 1999;107:59-64.

7. NHS UK. South Tees Hospitals. Cholinesterase. 2015. Available from: http://southtees.nhs.uk/services/pathology/tests/cholinesterase/

8. Gokani R, Jadav P, Shaikh N, et al. Serum cholinesterase as diagnostic marker of liver disease. IJBAR 2014:05.

9. Tureckey L, Kupcova V, Mojto V, et al. Serum cholinesterase activity and proteosynthetic function of liver in patients with diabetes mellitus. Bratisl Lek Listy 2005;106:266-9.

10. Sibae MR, Cappell MS. Accuracy of MELD scores in predicting mortality in decompensated cirrhosis from variceal bleeding, hepatorenal syndrome, alcoholic hepatitis, or acute liver failure as well as mortality after non-transplant surgery or TIPS. Dig Dis Sci 2011;56:977.

11. Yoo HY, Edwin D, Thuluvath PJ. Relationship of the model for end-stage liver disease (MELD) scale to hepatic encephalopathy, as defined by electroencephalography and europsychometric testing, and ascites. Am J Gastroenterol 2003;98:1395.

12. Weisinger RA. Laboratory tests in liver disease and approach to the patient with abnormal test. In: Goldman L, Bennett JC, eds. Cecil textbook of medicine. 21 st ed. Philadelphia: WB Saunders; 2000. pp. 775-777.

13. Whicher J, Spence C. When is serum albumin worth measuring? Ann Clin Biochem 1987;24:572-80.

14. Moss DW, Henderson AR. Clinical enzymology. In: Burits CA, Ashwood ER, eds. Tietz textbook of clinical chemistry. $2^{\text {nd }}$ ed. Philadelphia: WB Saunders; 1997. pp. 735-789.

15. Brown SS, Kalow W, Pilz W, et al. The plasma cholinesterases: a new perspective. Adv Clin Chem 1981;22:1-123.

16. Meng F, Yin X, Ma X, et al. Assessment 
of the value of serum cholinesterase as a liver function test for cirrhotic patients. Biomed Rep 2013;1:265-8.

17. Gu YJ, Zhong YB. Cholinesterase determination of evaluation of patients with liver cirrhosis of the liver reserves significance. Chin J Med Guide 2010;12:649-50.
18. Ramachandran J, Sajith KG, Priya S, et al. Serum cholinesterase is an excellent biomarker of liver cirrhosis. Trop Gastroenterol 2014;35:15-20. 